\title{
Evolutionary Multiobjective Optimization of a System-Level Motor Drive Design
}

\author{
Benjamin Cheong \\ Power Electronics, Machine and \\ Control Group (PEMC), \\ The University of Nottingham, UK \\ eexbc4@nottingham.ac.uk \\ Michael Galea \\ Power Electronics, Machine and \\ Control Group (PEMC), \\ The University of Nottingham, UK \\ ezzmg@nottingham.ac.uk
}

\author{
Paolo Giangrande \\ Power Electronics, Machine and \\ Control Group (PEMC), \\ The University of Nottingham, UK \\ ezzpg@nottingham.ac.uk \\ Pericle Zanchetta \\ Power Electronics, Machine and \\ Control Group (PEMC), \\ The University of Nottingham, UK \\ eezpz@nottingham.ac.uk
}

\author{
Xiaochen Zhang \\ Power Electronics, Machine and \\ Control Group (PEMC), \\ The University of Nottingham, UK \\ ezzxz2@nottingham.ac.uk \\ Patrick Wheeler \\ Power Electronics, Machine and \\ Control Group (PEMC), \\ The University of Nottingham, UK \\ eezpww@nottingham.ac.uk
}

\begin{abstract}
The use of optimization algorithms to design motor drive components is increasingly common. To account for component interactions, complex system-level models with many input parameters and constraints are needed, along with advanced optimization techniques. This paper explores the system-level optimization of a motor drive design, using advanced Evolutionary Multiobjective Optimization (EMO) algorithms. Practical aspects of their application to a motor drive design optimization are discussed, considering various modelling, search space definition, performance space mapping, and constraints handling techniques. Further, for illustration purposes, a motor drive design optimization case study is performed, and visualization plots for the design variables and constrained performances are proposed to aid analysis of the optimization results. With the increasing availability and capability of modern computing, this paper shows the significant advantages of optimization-based designs with EMO algorithms as compared to traditional design approaches, in terms of flexibility and engineering time.
\end{abstract}

Index Terms - Motor Drives, System analysis and design. Optimization methods, Permanent magnet machines, AC-AC power conversion

\section{INTRODUCTION}

The electric drive is an essential system in either industrial or mobile applications, since it handles the electromechanical power conversion [1, 2]. With a global push towards transportation electrification, there is an increasing need for effective ways to optimize its power density and efficiency.

Engineering designs can generally be broken down into two independent parts: modelling and optimization. Modelling techniques include the use of analytical equations, lumped parameter models, and numerical analysis, while optimization algorithms are further classified into two types: deterministic, where the algorithm searches for solutions systematically, and stochastic, where it explores the design space randomly [3].

Over the past decade, with drastic improvements in computing performance, different combinations of modelling techniques and optimization algorithms have been presented for the design of motor drive components, i.e. the machine and converter. Examples of deterministic optimization with analytical models can be found in $[4,5,6]$. Deterministic optimizations, however, are generally not computationally efficient for problems with many design variables if direct search approach is employed, due to an inherent 'step-size' problem, and they often get stuck in local optimums if solution gradients are used to speed up convergence [7]. In contrast, stochastic optimizations do not have a step-size problem and allow a finer scan in more promising regions compared to areas with fewer prospects. Further, they are gradient-free and do not hang on to local optimums, due to their use of random variables and a continually evolving population.

Evolutionary algorithms are inherently stochastic and examples of their use with analytical models for machine designs can be found in $[8,9,10,11]$, for converter designs in $[12,13]$, and for system-level motor drives in $[14,15]$. The optimization algorithms that feature in these publications are Genetic Algorithm (GA) [8, 12, 13], Particle Swarm Optimization (PSO) $[14,11]$, and Differential Evolution (DE) $[9,10]$. With the exception of [10], the mentioned publications do not consider the impact of design variables pre-selection, constraint handling techniques and performance space mapping methods on the optimization outcome. These aspects are key for an effective use of the optimization algorithms, especially for complex problems such as system-level motor 
drive optimization.

This work aims to present the use of modern EMO algorithms for the multiobjective optimization of a systemlevel motor drive design. Specific contributions of this paper are as follows:

- To discuss techniques for modelling, search space definition, performance space mapping, and constraint handling of a system-level motor drive optimization.

- To illustrate these concepts with a case study of motor drive optimization, specifically using the GDE3 algorithm, and to evaluate the results using new visualization methods that help their interpretation from an engineering standpoint.

Compared to previous publications, the work here is focused on performing large-scale system-level optimizations for a motor drive, where a high number of design variables are employed. Apart from addressing component modelling, practical aspects for the implementation of evolutionary algorithms to a motor drive design are discussed in detail here.

The rest of this paper is arranged as follows: in Section II, key concepts of a multiobjective optimization are defined, and in Section III, the basic principles of GDE3 are reviewed. Section IV provides several practical guidelines to the systemlevel modelling and implementation of optimization algorithms for motor drives. Section V presents a case study for a motor drive optimization using GDE3, visualization plots of the optimization outcome, and an engineering analysis of the observed trends. Finally, the conclusions of this paper are presented in Section VI.

\section{Multiobjective Optimization Problem}

This section defines several key concepts of multiobjective optimizations, as widely used in literature. Based on [7], a multiobjective optimization problem is defined as the problem of finding a set of input parameters $\vec{x}$ which minimizes a set of objective functions $\vec{f}(\vec{x})$ for an evaluation function $F$, subject to $o$ number of inequality constraints $g_{i}(\vec{x})$ and $p$ number of equality constraints $h_{j}(\vec{x})$. Mathematically, it is written as (1):

$$
\begin{array}{rlrl}
\text { find } \vec{x} & =\left[x_{1}, x_{2}, \cdots, x_{m}\right] & & \text { for } \vec{f}(\vec{x})=F(\vec{x}), \\
\text { such that } \vec{f}(\vec{x}) & =\min \left[f_{1}(\vec{x}), f_{2}(\vec{x}), \cdots, f_{n}(\vec{x})\right], \\
\text { subject to } g_{i}(\vec{x}) & \leq 0 & \text { for } i=\{1, \cdots, o\} \\
\text { and } h_{j}(\vec{x}) & =0 & \text { for } j=\{1, \cdots, p\}
\end{array}
$$

For multiobjective optimizations, there is often not one unique solution but a set of compromised solutions, also known as Pareto optimal solutions. The Pareto Optimality Theory [7] states that solutions $\vec{x}^{*}$ for a minimization problem are considered to be Pareto optimal, if there are no other feasible solutions $\vec{x}$ which would decrease an objective function without causing a simultaneous increase in at least another objective function. The objective functions of solutions $\vec{x}^{*}$ form the Pareto front, representing the best achievable performances.

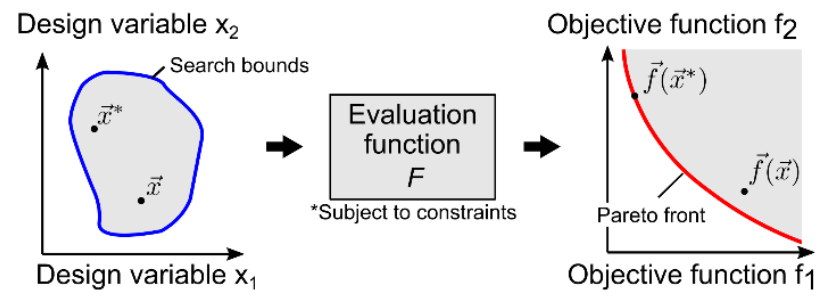

Fig. 1. Illustration of multiobjective optimization problem.

The problem search space is formed from all possible input parameter combinations and is mapped onto the performance space via an evaluation function, as illustrated in Fig. 1.

\section{EVOLUTIONARY ALGORITHMS}

Evolutionary algorithms are stochastic search techniques that mimic the natural selection process. This section reviews the basic principles of a relatively recent evolutionary algorithm, namely the Differential Evolution (DE).

DE was developed to be a reliable and versatile function optimizer that is also easy to use [16]. This algorithm shares many similarities with Genetic Algorithm (GA), in its use of a Parent and Child population, and bio-inspired operators to control the optimization. However, the main difference is that DE combines both crossover and mutation characteristics into one unique operator, which perturbs the current generation population members with scaled differences of randomly selected and distinct population members.

DE was originally designed for single objective problems, and to extend DE for multiobjective optimization problems, GDE3 is proposed in [17]. This algorithm uses a Pareto dominance concept for its selection, along with a crowding distance index to ensure a diverse set of solutions.

\section{PRACTICAL ASPECTS OF EMO APPLICATION}

As discussed before, the theoretical concept of multiobjective optimization and EMO algorithms can be applied for a system-level motor drive design, where optimal solutions are efficiently obtained. This section addresses practical aspects to their effective implementation.

\section{A. Modelling}

Optimization algorithms rely on a model for obtaining fitness values, implying that its success depends heavily on an accurate modelling of the motor drive. Using evolutionary algorithms, thousands of designs are typically evaluated per optimization run to solve high-dimensional problems. Thus, modelling speed is also an important criterion for system-level design optimizations with many design variables.

\section{B. $\quad$ Search Space Definition}

In a motor drive optimization-based design, input parameters are pre-selected to be either fixed as requirements, or modifiable as design variables. Each possible combination of design variables is subsequently allocated to a point in the 
search space. The pre-selection process for the design variables is often not straight-forward, and, therefore, four simple guidelines are provided as follows:

- Input parameters related to an external system should be fixed and determined by the application under study.

- Assumptions and limitations of the model implicitly restrict the variations of some input parameters, and, thus, eliminates them from being pre-selected as design variables.

- Compatibility of the optimization algorithm with the input parameter type should also be considered. The EMO algorithms described in the previous section are more suitable for continuous optimization problems, and they are less efficient if discrete input parameters are chosen as design variables.

- It is advantageous to have as many design variables as possible, to allow a wide range of solutions, even unconventional ones, to be evaluated. However, a compromise must be made, limited by the available computational resources.

Other important considerations when defining the search space include the selection of appropriate upper and lower boundaries for the design variables. It is important to ensure that the global optimum solutions are contained within these boundaries. Further, it is helpful to the algorithm's convergence speed, if search boundaries are chosen such that at least half of the search space contains feasible solutions. Fortunately, only crude estimates of these boundaries are needed with evolutionary algorithms.

\section{Performance Space Mapping}

Potential solutions from the search space are evaluated with the models, and their output or performances can be mapped onto a performance space. For motor drives, performance measures such as weight, losses, and cost are typically chosen as objective functions for minimization.

To identify the Pareto optimal solutions for a multiobjective problem, aggregate selection techniques, such as weighted sums method, are commonly used [11, 13]. In this approach, each objective function is multiplied with a weighting factor, and the solutions which share the lowest scalar weighted sum are considered to be Pareto optimal. Although this approach is easy to implement, it has a significant drawback of being unable to find optimum points on a non-convex Pareto front. Further, the choice of weights has a substantial impact on the optimality of the final design [7].

The alternative approach, employed in this work, is to directly incorporate the Pareto dominance concept into the selection operator of the optimization algorithm, thus removing the need for any weighting factors.

\section{Constraint Handling}

System requirements or limitations can be considered as constraints in the optimization if they are related to the output or performance of the model. There are several ways to handle these constraints in the evolutionary algorithm. The easiest way is the 'death penalty' method, where infeasible solutions are removed from the population. With this approach, the main limitation is that infeasible solutions do not contribute to the search for optimal solutions.

A more common approach is to add penalty functions to the objective functions of infeasible solutions, making them less attractive to the optimization, as compared to feasible solutions [9]. The challenge, however, is in tuning the penalty factors to relate the constraint violations to objective functions. If the penalty functions are too large, the search will struggle to explore the boundary between the feasible and infeasible region; if they are too small, too much time can be spent exploring the infeasible region.

To handle constraints without penalty factors, a constrained-domination rule can be directly incorporated into the selection operator of the evolutionary algorithm. The modified selection process is based on the following principles:

- If both individuals are feasible, the Pareto-dominance concept determines which individual is selected.

- If an individual is feasible but the other is not, the feasible individual is selected.

- If both individuals are infeasible, the individual with lower total constraint violation is selected.

In order to determine total constraint violation, individual constraint violations $c_{i}$ are first normalized as:

$$
c_{i}(\vec{x})=\begin{aligned}
& \min \left(1, g_{i, \max } / g_{i}(\vec{x})\right) \text { for max. constraint } g_{i, \max } \\
& \min \left(1, g_{i}(\vec{x}) / g_{i, \min }\right) \text { for min. constraint } g_{i, \min }
\end{aligned}
$$

Thus, $c_{i}(x)$ is 1 for feasible solutions, where constrained performance is smaller than the maximum limit (i.e. $g_{i}(x)<$ $g_{i, \max }$ ) or larger than the minimum limit (i.e. $g_{i}(x)>g_{i, \min }$ ), and $c_{i}(x)$ is larger than 1 for infeasible solutions [7]. The total constraint violation $c_{t o t}$ is the sum of individual constraint violations:

$$
c_{\text {tot }}(\vec{x})=\sum_{i=1}^{o} c_{i}(\vec{x})
$$

where $o$ is the total number of individual constraints. With the use of total constraint violation in (4) instead of maximum particular violation, no penalty factors are needed, and infeasible solutions are always quantitatively compared and penalized in a way such that they provide a search direction towards the feasible region [7].

\section{CASE Study: GDE3 Motor Drive Optimization}

This section presents a case study looking at using the GDE3 algorithm to optimize a motor drive system design, illustrating the concepts and theories discussed so far.

\section{A. Benchmark Motor Drive}

The benchmark for optimization in this case study is a motor 


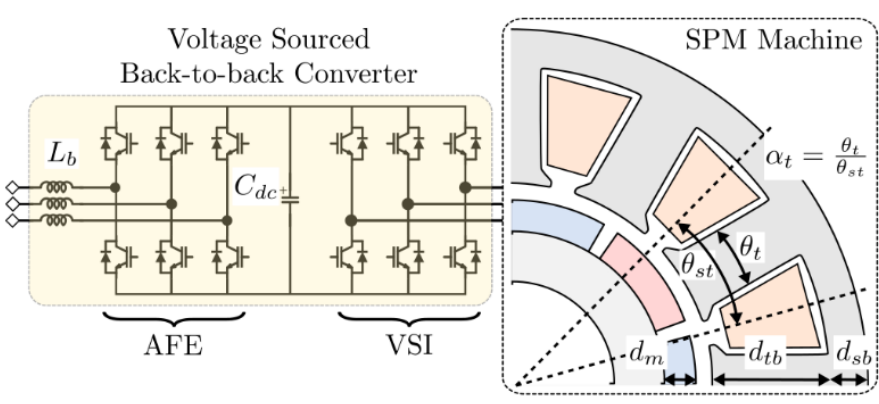

Fig. 2. Simplified diagram of motor drive considered.

(b)
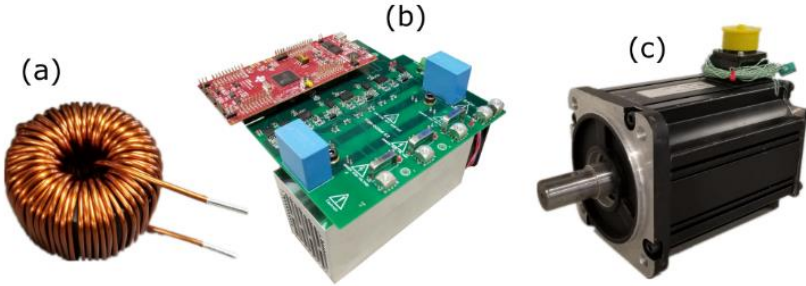

Fig. 3. Photos of prototype motor drive components: (a) toroidal core inductor, (b) two level voltage-source converter with DC-link capacitors and heatsink, (c) 12-slot 10-pole surface permanent magnet machine.

drive, which consists of a surface permanent magnet (SPM) machine, whose $\mathrm{d}$ - and q- axis inductances are equal [18], and voltage-source back-to-back converter (VSBBC), as is shown in Fig. 2. The SPM machine is a 12-slot 10-pole machine, rated at $1350 \mathrm{~W}$ and $1500 \mathrm{rpm}$, while the Active Front-End (AFE) rectifier and Voltage Source Inverter (VSI) are realized with Infineon IGBT4 FS50R12KT4 power modules, rated at 1200 $\mathrm{V}$ and $50 \mathrm{~A}$.

The boost inductors at the AFE input are $0.5 \mathrm{mH}$ toroidalcore single-layer inductors made of Mega Flux 60 core material (Chang Sung Corp.) rated at 15 A, while the DC-link capacitors are realized with two $0.25 \mathrm{mF}$ MKPB32778G polypropylene capacitors.

Analytical models are employed for the motor drive in the optimization, and details of their verifications can be found in $[19,20,21]$. While the cited papers detail only analytical models for the motor drive, this manuscript addresses their use in an optimization environment. Photos of the SPM machine prototype, voltage-source converters, and toroidal core inductors used for model verifications are shown in Fig. 3.

Unlike interior permanent magnet (IPM) machines where extra procedures are required to ensure geometrical feasibility of the rotors [22, 23], the SPM machine is easily parametrized according to the methods detailed in [24].

Analytical methods are computationally efficient, but they are generally unable to consider saturation effects in the magnetic core, unlike Finite Element (FE) methods. For initial stage design optimization and trade-off analysis, analytical models are often employed, considering the number of systemlevel design variables. At later design stages, more detailed component level optimizations can be performed by incorporating higher fidelity FE models in the design routine.
TABLE I

SElected Design Variables in CASE Study

\begin{tabular}{|c|c|c|c|}
\hline Design Variable & Range & Design Variable & Range \\
\hline \multicolumn{2}{|c|}{ For SPM Machine } & $d$-axis current, $i_{d o}(\mathrm{~A})$ & {$[-5,0]$} \\
\hline $\begin{array}{l}\text { Axial length, } \\
l(\mathrm{~mm})\end{array}$ & {$[30,80]$} & $q$-axis current, $i_{q o}(\mathrm{~A})$ & {$[8,18]$} \\
\hline $\begin{array}{l}\text { Turns in series per } \\
\text { phase, } N_{t} \text { (turns) }\end{array}$ & {$[15,45]$} & \multicolumn{2}{|c|}{ For VSBBC Converter } \\
\hline $\begin{array}{l}\text { Wire diameter, } w_{\text {dia }} \\
(\mathrm{mm})\end{array}$ & {$[0.5,0.9]$} & $\begin{array}{l}\text { DC-link voltage, } \\
V_{d c}(\mathrm{~V})\end{array}$ & $\begin{array}{l}{[100,} \\
300]\end{array}$ \\
\hline $\begin{array}{l}\text { Depth of magnet, } \\
d_{m}(\mathrm{~mm})\end{array}$ & {$[2,20]$} & $\begin{array}{l}\text { VSI switching freq., } \\
f_{s w v}(\mathrm{kHz})\end{array}$ & {$[4,20]$} \\
\hline $\begin{array}{l}\text { Depth of stator } \\
\text { tooth, } d_{t b}(\mathrm{~mm})\end{array}$ & {$[5,25]$} & AFE switching freq., & {$[4,20]$} \\
\hline $\begin{array}{l}\text { Depth of stator } \\
\text { back-iron, } d_{\text {sh }}(\mathrm{mm})\end{array}$ & {$[4,10]$} & $\begin{array}{l}\text { Boost inductance, } \\
L_{b}(\mathrm{mH})\end{array}$ & {$[0.2,0.8]$} \\
\hline $\begin{array}{l}\text { Stator tooth fraction, } \\
\alpha_{t} \text { (p.u.) }\end{array}$ & {$[0.4,0.8]$} & $\begin{array}{l}\text { DC-link capacitance, } \\
C_{d c}(\mathrm{mF})\end{array}$ & {$[0.2,2]$} \\
\hline
\end{tabular}

TABLE II

Constraints ENFORCED in CASE STUdy

\begin{tabular}{lcll}
\hline \multicolumn{1}{c}{ Constraint } & Value & \multicolumn{1}{c}{ Constraint } & Value \\
\hline $\begin{array}{l}\text { Max. slot packing } \\
\text { factor, } k_{p f, \max } \text { (p.u.) }\end{array}$ & 0.723 & $\begin{array}{l}\text { Max. stator back-iron } \\
\text { flux density, } B_{s b}(\mathrm{~T})\end{array}$ & 1.15 \\
$\begin{array}{l}\text { Min. output torque, } \\
\tau_{e, \text { min }}(\mathrm{Nm})\end{array}$ & 8.52 & $\begin{array}{l}\text { Max. VSI modulation } \\
\text { index, } M_{v} \text { (p.u.) }\end{array}$ & 1.15 \\
$\begin{array}{l}\text { Max. magnet } \\
\text { temperature, } T_{p m}\left({ }^{\circ} \mathrm{C}\right)\end{array}$ & 58.3 & $\begin{array}{l}\text { Max. AFE modulation } \\
\text { index, } M_{a} \text { (p.u.) }\end{array}$ & 1.15 \\
$\begin{array}{l}\text { Max. stator winding } \\
\text { temperature, } T_{w}\left({ }^{\circ} \mathrm{C}\right)\end{array}$ & 71.4 & $\begin{array}{l}\text { Max. input pk-pk current } \\
\text { ripple, } \Delta i_{p p}(\%)\end{array}$ & 26.1 \\
$\begin{array}{l}\text { Max. stator tooth flux } \\
\text { density, } B_{s t}(\mathrm{~T})\end{array}$ & 1.47 & $\begin{array}{l}\text { Max. DC-link voltage } \\
\text { overshoot, } V_{d c o}(\%)\end{array}$ & 1.22 \\
\hline
\end{tabular}

\section{B. Optimization Setup}

In this case study, two objective functions are considered: total weight $w_{t o t}$ and total losses $P_{l, t o t}$ of the motor drive, since they are perceived as high priority at the preliminary design stage. The total weight considers the active weight of the machine (i.e. stator and rotor cores, windings, permanent magnets, etc.), and weight of the converter (i.e. heatsink, inductors, and capacitors), while total losses consist of the machine copper and iron losses, and converter semiconductor losses. Provided that the relevant models are available, other objectives such as cogging torque and cost can also be evaluated, based on requirements as demanded by the project.

A total of 14 design variables are chosen from a combined set of machine and converter input parameters, and they are listed along with their boundaries, in Table I. For the machine parameters, depth of magnet, stator tooth, stator back-iron, and stator tooth fraction variables can be found from the dimensional drawing in Fig. 2. For this particular study, the number of strands per winding turn is fixed at 6 , since a higher number would increase the manufacturing complexity, while wire diameter and number of turns are selected as design variables. The design variable boundaries are chosen based on the benchmark motor drive's parameters.

Meanwhile, ten design constraints, as listed in Table II, are imposed on the output performances to ensure that the design 


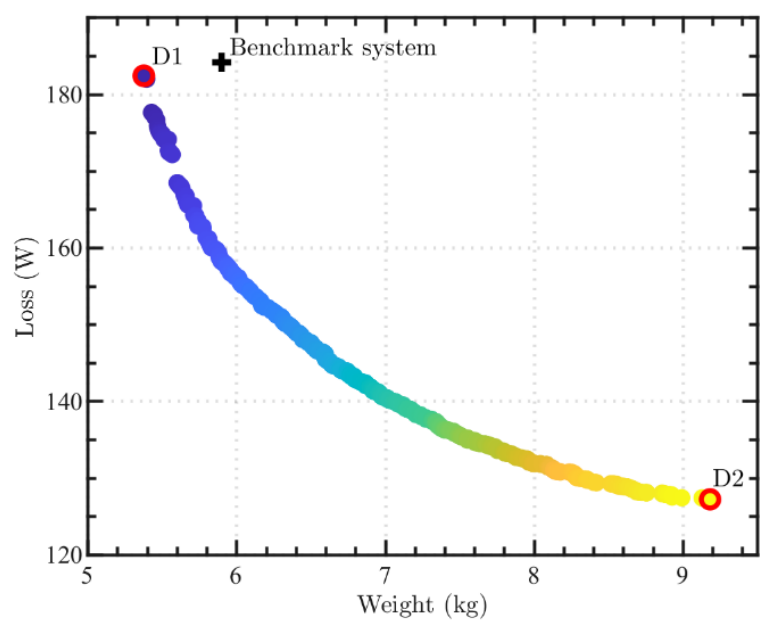

Fig. 4. Pareto front solutions for a motor drive system-level optimization; D1 and D2 design solutions are selected for further software verifications.

solutions are feasible and that they comply with requirements, i.e. temperature limits are not exceeded to avoid thermal aging [25], magnetic materials are not saturated, etc.

For a fair comparison of the un-optimized benchmark design against the optimized designs, their constrained performances are fixed to be the same. This means that the new designs' output performances must match the benchmark motor drive output performances, in terms of torque, winding temperatures, current ripple and voltage overshoot magnitudes, etc.

The GDE3 algorithm employed in this case study is set up with a crossover rate and scaling factor of 0.2 and 0.5 , respectively, based on recommendations from [17]. Other values for the crossover rate and scaling factors have also been tested and the algorithm's convergence rate is found to be sensitive to these parameters. However, detailed tuning of the parameters is not considered within the scope of this work.

For each optimization run, a large population size is chosen with $\mathcal{N}=200$ per design variable, and the optimization is stopped after a maximum number of $g_{\max }=2000$ generations. Other stopping criterions include an adaptive approach where optimization is stopped when no further improvements are detected. In this work, only the maximum number of generations criterion is employed.

The motor drive analytical models and optimization algorithm are coded entirely in Matlab for ease of integration. With code vectorization for efficiency, the system-level model takes approximately $3 \times 10^{-2}$ seconds to run on a normal PC. Besides, the Matlab Parallel Computing toolbox allows the use of multicore desktops to speed up the evaluation process incredibly. Calculations for this case study are performed using the University of Nottingham High-Performance Computing (HPC) facility, on computer nodes that are equipped with $2 \times 20$ cores Intel Xeon Gold 6138 processors. For an optimization run with 14 design variables, a total of $14 \times 200 \times 2000=5.6$ million evaluations can be completed in less than 1.5 hours.

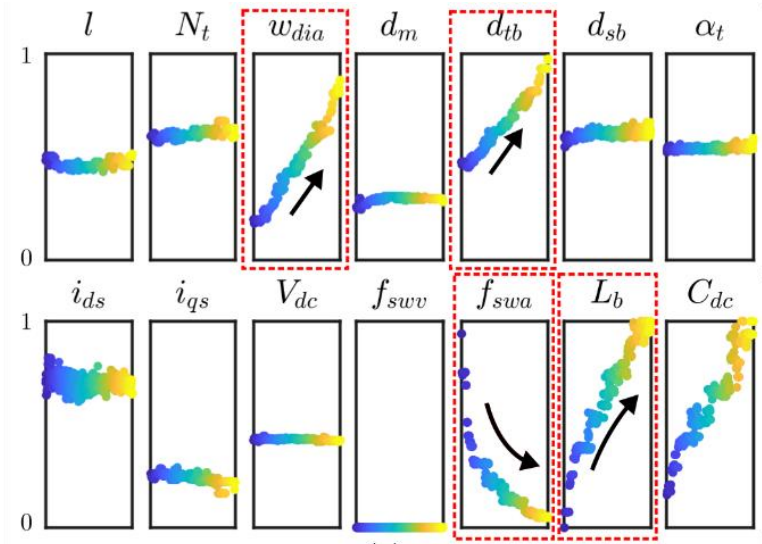

(a)

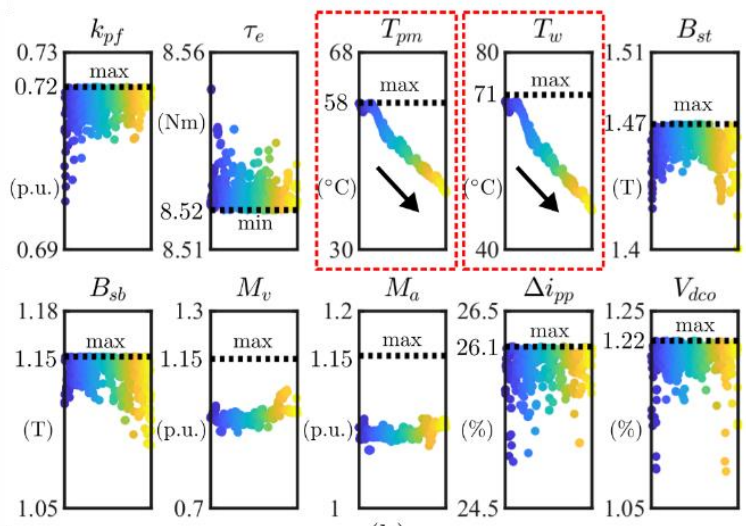

(b)

Fig. 5. (a) Normalized design variables (per unit) and (b) Constrained performances of Pareto front solutions for system-level optimization.

\section{Analysis of Optimization Results}

The Pareto front results from the optimization is plotted in Fig. 4. The results show that a wide range of design solutions are found, offering significant improvements in weight and losses over the benchmark design.

A general criticism of optimization-based designs is that it is easy to lose track of the optimization process and, hence, not gain useful engineering insights from the design. For improved clarity and visibility, two supplementary plots are proposed, to visualize the process and observe design trends:

- Design variable plot in Fig. 5a, showing the design variables for the Pareto front solutions normalized to the upper and lower limits of their search space.

- Constraint plot in Fig. 5b, showing the constrained performance outputs for the Pareto front solutions.

In both these plots, the lower weight Pareto front individuals are placed towards the left of each window, while lower loss individuals on the right. As can be seen from the constraint plot, all Pareto front solutions are shown to meet the constrained performance requirements.

From the design variable plot, a clear trend of increasing stator tooth depth $d_{t b}$ and wire diameter $w_{d i a}$ is observed for lower loss solutions. As number of turns remained almost the 
same, this increasing trend can be explained by lower winding resistances due to larger wire diameter and cross-sectional areas. Thus, a larger slot area, achieved by increasing $d_{t b}$ is needed to accommodate the windings, subject to a maximum slot packing factor constraint $k_{p f \text {, max }}$. Finally, this results in an increased machine diameter, larger outer surface and improved cooling performance, and, thus, explaining the lower winding and magnet temperatures in Fig. $5 \mathrm{~b}$.

Furthermore, the inverse relationship between AFE switching frequency against losses and boost inductance can also be seen. For lower loss solutions, the AFE switching frequency is reduced to decrease switching losses, but at the same time, boost inductance and DC-link capacitance must increase, to ensure that the input current ripple $\Delta i_{p p}$ and DClink voltage overshoot $V_{d c o}$ constraints are not violated. Due to these constraints, the optimal DC-link voltages for the Pareto front solutions is seen to be almost constant.

It is interesting to also observe some negative d-axis fieldweakening current $i_{d}$ in the Pareto front solutions. This is because in this case, a small amount of field-weakening is found to allow converter DC-link voltage to be reduced for a given machine operating point, reducing converter side switching losses, which lead to an overall reduction in motor drive total losses.

Finally, from the design variable plot, it can be observed that without any current ripple requirements between the VSI and motor, the optimization automatically favors using a minimal converter switching frequencies to reduce switching losses.

\section{Verification of Design Solutions}

To assess the optimization results' accuracy and validity, specific design solutions from the Pareto front of the motor drive system-level optimization are selected for further verifications using $\mathrm{FE}$ and time-domain simulation software. The FE tool selected for this analysis is Infolytica Magnet [26], while the time-domain simulation tool is PLECS.

From the Pareto front solutions, the lowest weight solution (D1) and lowest loss solution (D2), as indicated in Fig. 4, are selected. Their corresponding design variables, constrained performances, and objective functions (calculated using the analytical model) are tabulated in Table III.

At the rated design operating point, the 2D-FE simulation models for the design solutions D1 and D2 are shown in Fig. 6 , in comparison with the benchmark solution. It can be seen from the figure that D2 has larger slot areas, to accommodate wires of larger diameters. On the other hand, D1, despite having a similar outer diameter and length to the benchmark design, has thicker magnets and smaller slot areas. Machine performances calculated from the FE simulation are summarized in Table IV and are shown to match well with the analytical predictions.

PLECS time-domain simulations allows semiconductor losses and converter transient performances to be predicted. The simulated performances are also compared against the

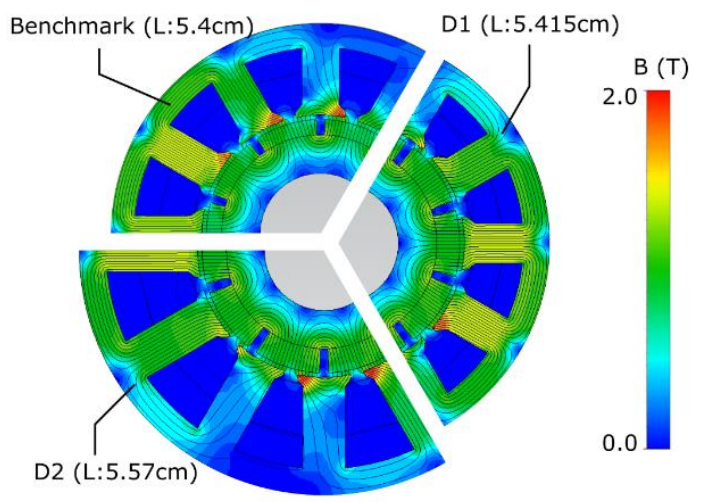

Fig. 6. 2D-finite element analysis under rated operating conditions for (a) Benchmark, (b) D1, and (c) D2 design solutions.

(a)
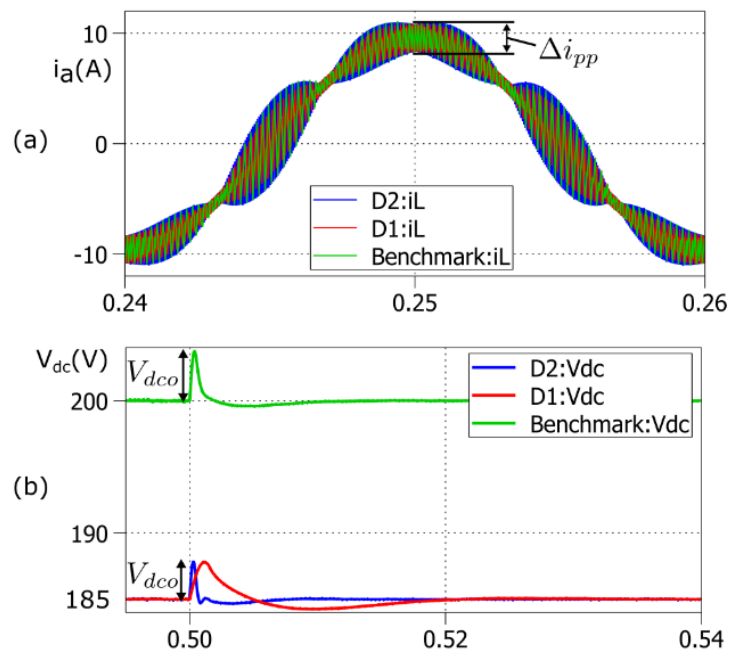

Fig. 7. PLECS simulation results for (a) input phase- $a$ current under rated load conditions; and (b) DC-link voltage overshoot during worst-case load transient at $\mathrm{t}=0.5 \mathrm{~s}$.

analytical predictions in Table IV, showing an accurate match. Meanwhile, Fig. 7 shows how the current ripples and DC-link voltage overshoots are calculated from the simulated converter waveforms. As can be seen from Fig. 7a and 7b, all three solutions have the same current ripples magnitude and voltage overshoot percentage. The worst-case load transient is assumed here to be an externally triggered step removal of rated load from the converter output.

In practice, a designed system will unlikely fall directly on the Pareto front as desired and the design solutions should be subject to extra experimental verifications. This would also give the designer an opportunity to accurately evaluate and quantify the mismatch between designed/optimized and manufactured electric drives. However, given that the employed analytical models have already been experimentally verified in $[19,20,21]$ and considering only an preliminary stage design optimization, only software verifications are performed and discussed in this work.

\section{E. Further Evaluations}

Often, a higher-level power system architecture designer 
TABLE III

PARAMETERS OF D1 (LOWEST WEIGHT) AND D2 (LOWEST LOSS)

\begin{tabular}{|c|c|c|c|c|c|c|c|}
\hline Symbol & Bench. & D1 & D2 & Symbol & Bench. & D1 & D2 \\
\hline \multicolumn{4}{|c|}{ Design Variables } & \multicolumn{4}{|c|}{ Constraints } \\
\hline$l(\mathrm{~mm})$ & 54.0 & 54.1 & 55.7 & $k_{p f}(-)$ & 0.72 & 0.71 & 0.72 \\
\hline$N_{t}(-)$ & 30 & 33 & 33 & $\tau_{e}(\mathrm{Nm})$ & 8.52 & 8.52 & 8.52 \\
\hline$w_{d i a}(\mathrm{~mm})$ & 0.68 & 0.58 & 0.85 & $T_{p m}\left({ }^{\circ} \mathrm{C}\right)$ & 71.4 & 70.1 & 47.9 \\
\hline$d_{m}(\mathrm{~mm})$ & 4.4 & 6.6 & 7.1 & $T_{w}\left({ }^{\circ} \mathrm{C}\right)$ & 58.3 & 58.2 & 40.9 \\
\hline$d_{t b}(\mathrm{~mm})$ & 17.0 & 14.5 & 24.5 & $B_{s t}(\mathrm{~T})$ & 1.47 & 1.46 & 1.40 \\
\hline$d_{s b}(\mathrm{~mm})$ & 6.88 & 7.60 & 7.80 & $B_{s b}(\mathrm{~T})$ & 1.15 & 1.12 & 1.10 \\
\hline$\alpha_{t}(-)$ & 0.60 & 0.61 & 0.64 & $M_{v}(-)$ & 0.76 & 0.97 & 0.99 \\
\hline$i_{d o}(\mathrm{~A})$ & 0 & -1.49 & -1.75 & $M_{a}(-)$ & 1.00 & 1.08 & 1.08 \\
\hline$i_{q o}(\mathrm{~A})$ & 13.2 & 10.6 & 10.2 & $\Delta i_{p p}(\%)$ & 26.2 & 25.7 & 26.1 \\
\hline$V_{d c}(\mathrm{~V})$ & 200 & 185 & 185 & $V_{d c o}(\%)$ & 1.22 & 1.20 & 1.13 \\
\hline$f_{s w v}(\mathrm{kHz})$ & 10 & 4 & 4 & \multicolumn{4}{|c|}{ Objective Functions } \\
\hline$f_{s w a}(\mathrm{kHz})$ & 10 & 19 & 4.8 & $w_{\text {tot }}(\mathrm{kg})$ & 5.90 & 5.38 & 9.91 \\
\hline$L_{b}(\mathrm{mH})$ & 0.5 & 0.2 & 0.8 & $P_{l, t o t}(\mathrm{~W})$ & 184 & 182 & 130 \\
\hline$C_{d c}(\mathrm{mF})$ & 0.5 & 0.49 & 2.0 & & & & \\
\hline
\end{tabular}

TABLE IV

SIMULATION RESULTS OF D1 (LOWEST WEIGHT) AND D2 (LOWEST LOSS)

\begin{tabular}{|c|c|c|c|c|c|c|}
\hline \multirow[t]{2}{*}{ Output/Performance } & \multicolumn{2}{|c|}{ Benchmark } & \multicolumn{2}{|c|}{ D1 } & \multicolumn{2}{|c|}{ D2 } \\
\hline & Ana. & Sim. & Ana. & Sim. & Ana. & Sim. \\
\hline & \multicolumn{6}{|c|}{ Finite-Element Analysis } \\
\hline Phase resistance $(\mathrm{m} \Omega)$ & 202.5 & 203.0 & 312.2 & 313.1 & 152.9 & 153.3 \\
\hline Inductance $(\mathrm{mH})$ & 2.25 & 2.27 & 2.69 & 2.52 & 2.86 & 2.81 \\
\hline Back EMF (V) & 48.57 & 47.96 & 60.97 & 60.10 & 63.95 & 62.98 \\
\hline \multirow[t]{2}{*}{ Output torque $(\mathrm{Nm})$} & 8.53 & 8.41 & 8.52 & 8.57 & 8.52 & 8.61 \\
\hline & \multicolumn{6}{|c|}{ Time-domain Simulation Analysis } \\
\hline VSI total losses (W) & 52.79 & 53.64 & 28.27 & 29.76 & 27.23 & 26.04 \\
\hline AFE total losses (W) & 38.08 & 37.05 & 55.75 & 53.26 & 25.49 & 24.80 \\
\hline $\begin{array}{l}\text { Max. input pk-pk } \\
\text { current ripple (A) }\end{array}$ & 2.50 & 2.44 & 2.46 & 2.52 & 2.46 & 2.58 \\
\hline $\begin{array}{l}\text { Max. DC-link voltage } \\
\text { overshoot (V) }\end{array}$ & 202.4 & 203.73 & 186.9 & 187.85 & 186.8 & 187.8 \\
\hline
\end{tabular}

will be interested to also consider the effects of varying the system input requirements on the motor drive design. Two examples are provided as follows to demonstrate the use of optimization for this purpose.

\section{1) Effect of Machine Operating Points}

In many motor drive systems, the electrical machine is connected to a mechanical drive train system via a gearbox [27]. The gearbox transmission ratio defines the machine operating point and is typically chosen at a system architecture level.

Given different machine operating points, the optimization setup reveals a different set of Pareto front solutions, as shown in Fig. 8. For the same search space boundaries and constrained performances, a comparison between the Pareto front results shows that, for the same output power, high-speed systems are lighter in terms of weight, as expected, due to their lower output torque requirements. However, they are shown to be more limited in terms of efficiency. These observations can be explained by considering the design variable plot and constrained performances plot in Fig. 9a and b, respectively.

It is observed in Fig. 9a, that optimal winding currents $i_{d s}$ and $i_{q s}$ vary only by a little for designs with different operating

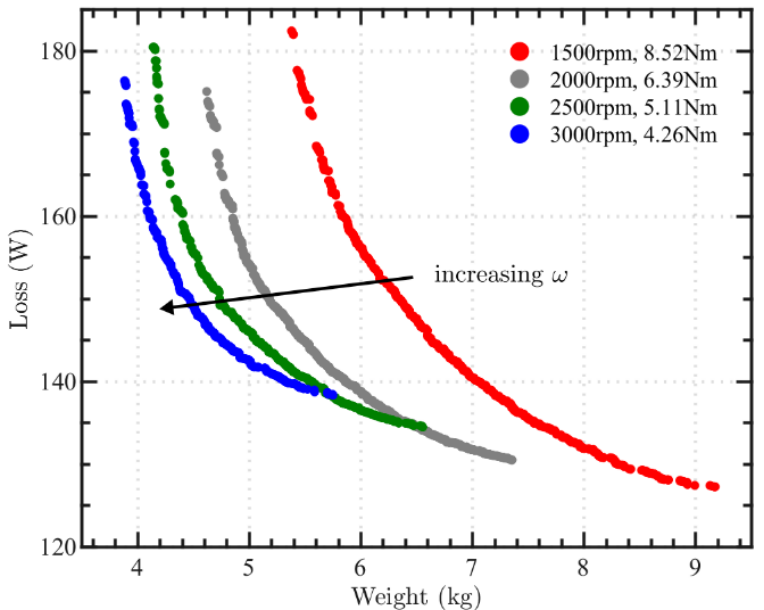

Fig. 8. Pareto front solutions for system-level optimizations with different machine operating points.

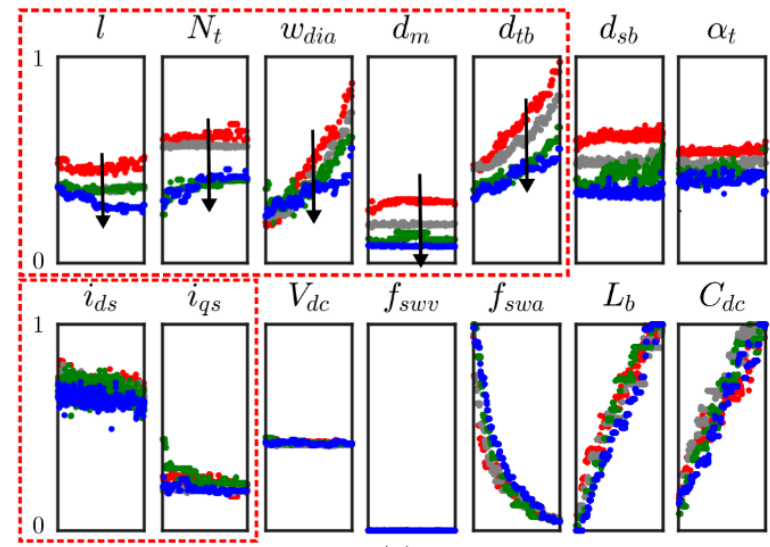

(a)

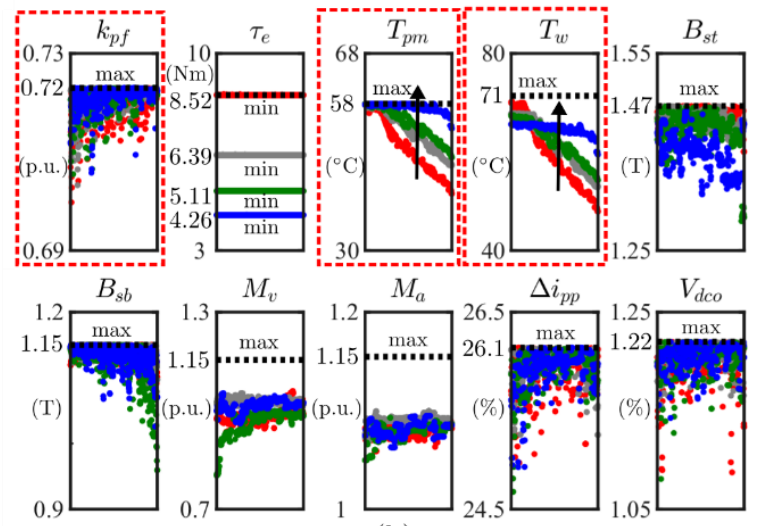

(b)

Fig. 9. (a) Normalized design variables (per unit) and (b) constrained performances of Pareto front solutions for system-level optimizations with different machine operating points.

point requirements. On the other hand, significant changes are seen for the machine geometry and number of winding turns. This indicates that although machine torque $\tau_{e}$ is proportional to both $i_{q}$ and machine flux linkage $\psi_{m}$ components, the optimization algorithm favors reducing $\psi_{m}$ over $i_{q}$. This is mainly due to the geometrical and thermal constraints of the system, which require the system solutions to maintain the 


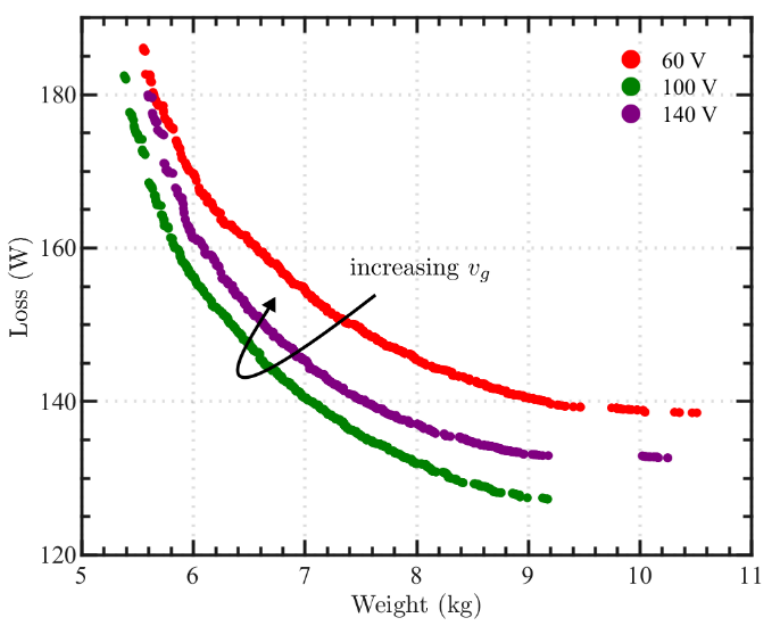

Fig. 10. Pareto front solutions for system-level optimizations with different AC input grid voltages.

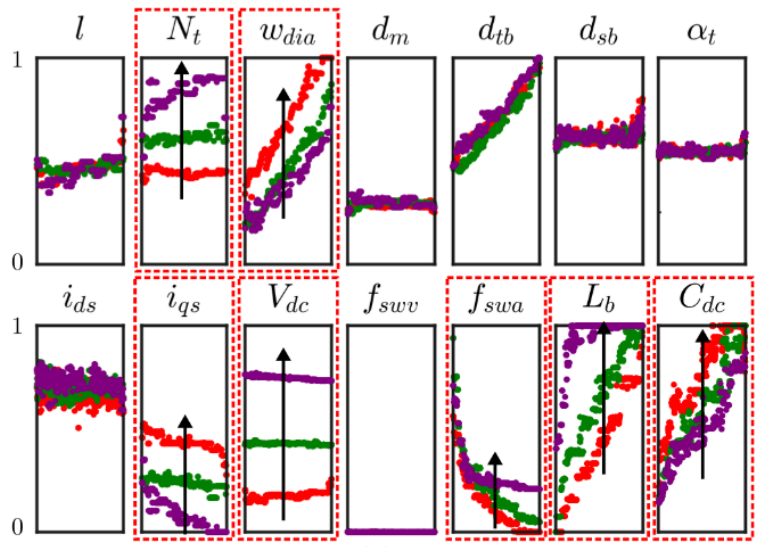

(a)

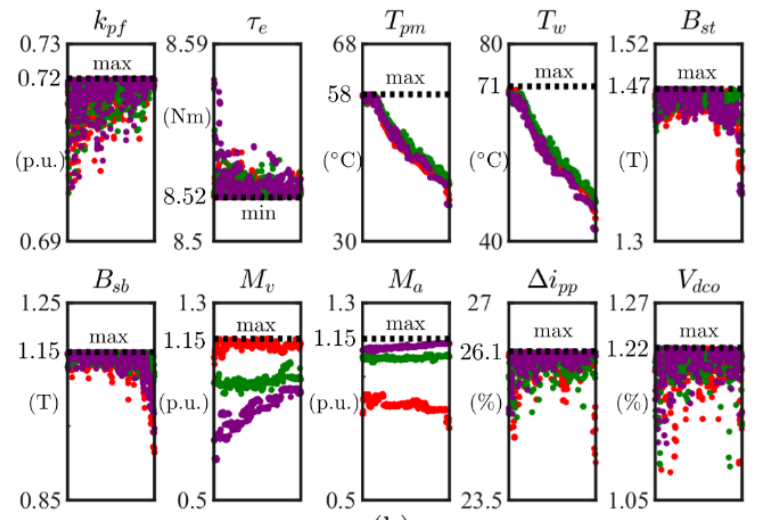

(b)

Fig. 11. (a) Normalized design variables (per unit) and (b) constrained performances of Pareto front solutions for system-level optimizations with different AC input grid voltages.

same machine packing factor $k_{p f}$ and temperatures $\left(T_{p m}\right.$ and $\left.T_{w}\right)$ as the benchmark system.

\section{2) Effect of AC Grid Input Voltage}

At a power system architecture level, the AC grid input voltage to the motor drive is also typically optimized beforehand and provided to the designer as input requirements.
With this optimization setup, the effect of selecting different AC grid input voltage can be also evaluated. As an example, Pareto fronts resulting from different input voltages in this case study are shown in Fig. 10. In this figure, it is shown that neither increasing or decreasing the AC grid input voltage resulted in better weight and efficiency performances.

From the design variable plot and constrained performance plot in Fig. 11a and b, respectively, the optimal converter DClink voltages are significantly influenced by the choice of the $\mathrm{AC}$ input voltage. This subsequently changes the optimal machine voltage, which is proportional to its number of turns and magnetic flux linkage.

For motor drive systems with an input grid voltage $v_{g}$ of $60 \mathrm{~V}$, the DC-link voltage $V_{d c}$ needs to be reduced, due to constraints on the input current ripples, which is proportional to the difference in $V_{d c}$ and $v_{g}$. As a result, the machine's terminal voltage and magnetic flux linkage are limited, subject to the maximum VSI modulation index constraint. Thus, higher output currents are seen at both its input and output, and thus the system suffers from high conduction losses in the machine and converter.

Similarly, if an input grid voltage of $140 \mathrm{~V}$ is used, the DClink voltage needs to be increased, due to the input current ripples constraints. Consequently, higher switching losses are obtained in the converter.

Finally, as input grid voltage and, subsequently, DC-link voltage is increased, the input current is decreased. As input current ripple constraint is enforced as a fixed percentage of the fundamental current component, the allowable current ripple magnitude is also reduced. Thus, larger boost inductances $L_{b}$ are needed to provide the required attenuation.

\section{CONCLUSIONS}

The use of evolutionary algorithms is very attractive for the multiobjective optimization of motor drives, due to their ability to handle complex problems with many design variables, constraints, and objectives. This paper has reviewed the basic principles of evolutionary optimization algorithms, considering specifically the GDE3 variant, and provided practical guidelines for its application to a motor drive design optimization. These concepts were further illustrated using a motor drive optimization case-study. In addition, visualization plots for the optimization variables and constrained performances have been proposed to extract useful engineering insights from the optimization outcome.

With the increasing availability and capability of modern computing, there is clearly a growing potential for the use of EMO algorithms in optimization-based motor drive designs. The success of this design approach, however, relies heavily on having models at the right fidelity level and a proper implementation of the algorithms. 


\section{ACKNOWLEDGMENT}

This project has received funding from the Clean Sky 2 Joint Undertaking under the European Union's Horizon 2020 research and innovation programme under grant agreement no. 807081.

\section{REFERENCES}

[1] V. Madonna, P. Giangrande and M. Galea, "Electrical Power Generation in Aircraft: Review, Challenges, and Opportunities," IEEE Transactions on Transportation Electrification, vol. 4, pp. 646-659, 92018.

[2] M. Lukic, P. Giangrande, A. Hebala, S. Nuzzo and M. Galea, "Review, Challenges, and Future Developments of Electric Taxiing Systems," IEEE Transactions on Transportation Electrification, vol. 5, pp. 1441-1457, 122019.

[3] G. Bramerdorfer, J. A. Tapia, J. J. Pyrhonen and A. Cavagnino, "Modern Electrical Machine Design Optimization: Techniques, Trends, and Best Practices," IEEE Transactions on Industrial Electronics, vol. 65, pp. 7672-7684, 102018.

[4] C. Gammeter, Y. Drapela, A. Tuysuz and J. W. Kolar, "Weight optimization of a machine for airborne wind turbines," in IECON 2014 - 40th Annual Conference of the IEEE Industrial Electronics Society, 2014.

[5] Q. Wang, X. Zhang, R. Burgos, D. Boroyevich, A. M. White and M. Kheraluwala, "Design and Implementation of a Two-Channel Interleaved Vienna-Type Rectifier With >99\% Efficiency," IEEE Transactions on Power Electronics, vol. 33, pp. 226-239, 12018.

[6] R. M. Burkart and J. W. Kolar, "Comparative eta-rho-sigma Pareto Optimization of Si and SiC Multilevel Dual-Active-Bridge Topologies With Wide Input Voltage Range," IEEE Transactions on Power Electronics, vol. 32, pp. 5258-5270, 72017.

[7] C. A. Coello, D. A. Veldhuizen and G. B. Lamont, Evolutionary Algorithms for Solving Multi-Objective Problems, Springer-Verlag $\mathrm{GmbH}, 2007$.

[8] B. N. Cassimere and S. D. Sudhoff, "Population-Based Design of Surface-Mounted Permanent-Magnet Synchronous Machines," IEEE Transactions on Energy Conversion, vol. 24, pp. 338-346, 6 2009.

[9] Y. Duan and D. M. Ionel, "A Review of Recent Developments in Electrical Machine Design Optimization Methods With a Permanent-Magnet Synchronous Motor Benchmark Study," IEEE Transactions on Industry Applications, vol. 49, pp. 1268-1275, 5 2013.

[10] A. Fatemi, D. M. Ionel, N. A. O. Demerdash and T. W. Nehl, "Fast Multi-Objective CMODE-Type Optimization of PM Machines Using Multicore Desktop Computers," IEEE Transactions on Industry Applications, vol. 52, pp. 2941-2950, 72016.

[11] S. G. Min, G. Bramerdorfer and B. Sarlioglu, "Analytical Modeling and Optimization for Electromagnetic Performances of Fractional-Slot PM Brushless Machines," IEEE Transactions on Industrial Electronics, vol. 65, pp. 4017-4027, 52018.

[12] M. Mirjafari, S. Harb and R. S. Balog, "Multiobjective Optimization and Topology Selection for a Module-Integrated Inverter," IEEE Transactions on Power Electronics, vol. 30, pp. 4219-4231, 82015.

[13] M. DAntonio, C. Shi, B. Wu and A. Khaligh, "Design and Optimization of a Solar Power Conversion System for Space Applications," IEEE Transactions on Industry Applications, vol. 55, pp. 2310-2319, 52019.

[14] M. Rottach, P. W. Wheeler and C. Gerada, "Design Optimisation of a Fault-Tolerant PM Motor Drive for an Aerospace Actuation Application," in 7th IET International Conference on Power Electronics, Machines and Drives (PEMD 2014), 2014.

[15] D. Zarko, M. Kovacic, S. Stipetic and D. Vuljaj, "Optimization of electric drives for traction applications," in 2017 19th International Conference on Electrical Drives and Power Electronics (EDPE), 2017.

[16] K. V. Price, J. Lampinen and R. Storn, Differential Evolution, Springer-Verlag, 2005.

[17] S. Kukkonen and J. Lampinen, "GDE3: The third Evolution Step of Generalized Differential Evolution," in IEEE Congress on Evolutionary Computation, 2005.

[18] P. Giangrande, S. V. Bozhko, C. I. Hill and C. Gerada, "A Novel Multi-Level Electro-Mechanical Actuator Virtual Testing and Analysis Tool," in 7th IET International Conference on Power Electronics, Machines and Drives (PEMD 2014), 2014.

[19] B. Cheong, P. Giangrande, X. Zhang, P. Wheeler, P. Zanchetta and M. Galea, "Fast and Accurate Model for Optimization-based Design of Fractional-Slot Surface PM Machines," in The 22nd international Conference on Electrical Machines and Systems (ICEMS 2019), 2019.

[20] B. Cheong, P. Giangrande, X. Zhang, P. Wheeler, P. Zanchetta and M. Galea, "Fast and Accurate Multi-Physics Model for Optimization-based Design of VSBBC," in IECON 2019 - 45th Annual Conference of the IEEE Industrial Electronics Society, 2019.

[21] B. Cheong, P. Giangrande, X. Zhang, P. Wheeler, P. Zanchetta and M. Galea, "System Level Motor Drive Modelling for Optimization-based Designs," in 201921 st European Conference on Power Electronics and Applications (EPE'19 ECCE Europe), 2019.

[22] S. Stipetic, W. Miebach and D. Zarko, "Optimization in design of electric machines: Methodology and workflow," in 2015 Intl Aegean Conference on Electrical Machines \& Power Electronics (ACEMP), 2015 Intl Conference on Optimization of Electrical \& Electronic Equipment (OPTIM) \& 2015 Intl Symposium on Advanced Electromechanical Motion Systems (ELECTROMOTION), 2015.

[23] D. Zarko, S. Stipetic, M. Martinovic, M. Kovacic, T. Jercic and Z. Hanic, "Reduction of Computational Efforts in Finite ElementBased Permanent Magnet Traction Motor Optimization," IEEE Transactions on Industrial Electronics, vol. 65, pp. 1799-1807, 2 2018.

[24] S. D. Sudhoff, Power Magnetic Devices, S. D. Sudhoff, Ed., John Wiley \& Sons, 2014.

[25] V. Madonna, P. Giangrande, L. Lusuardi, A. Cavallini, C. Gerada and M. Galea, "Thermal Overload and Insulation Aging of Short Duty Cycle, Aerospace Motors," IEEE Transactions on Industrial Electronics, vol. 67, pp. 2618-2629, 42020.

[26] S. A. Odhano, P. Giangrande, R. Bojoi and C. Gerada, "Selfcommissioning of interior permanent magnet synchronous motor drives with high-frequency current injection," in 2013 IEEE Energy Conversion Congress and Exposition, 2013.

[27] C. Sciascera, P. Giangrande, C. Brunson, M. Galea and C. Gerada, "Optimal design of an electro-mechanical actuator for aerospace application," in IECON 2015 - 41 st Annual Conference of the IEEE Industrial Electronics Society, 2015. 